\title{
Introducción de la Perspectiva de Género en la docencia de Ingeniería del Software Introduction of the Gender Perspective in the Teaching of Software Engineering
}

\author{
Alicia García-Holgado ${ }^{1}$, Francisco J. García-Peñalvo ${ }^{1}$, Juanjo Mena², Carina González ${ }^{3}$ \\ aliciagh@usal.es,fgarcia@usal.es, juanjo_mena@usal.es, cjgonza@ull.edu.es
}

\author{
${ }^{1}$ Dpto. de Informática y Automática \\ Grupo de Investigación GRIAL \\ Instituto Universitario de Ciencias de la \\ Educación \\ Universidad de Salamanca \\ Salamanca, España
}

\author{
${ }^{2}$ Dpto. de Didáctica, Organización y \\ Métodos de Investigación \\ Grupo de Investigación Innovación en \\ Tecnología Educativa (GITE) \\ Universidad de Salamanca \\ Salamanca, España
}

\author{
${ }^{3}$ Dpto. de Ingeniería Informática y de \\ Sistemas \\ Instituto Universitario de Estudios de \\ las Mujeres (IUEM) \\ Universidad de la Laguna \\ San Cristobal de la Laguna, Santa Cruz \\ de Tenerife, España
}

\begin{abstract}
Resumen- La desigualdad de género es un problema a nivel mundial, pero se encuentra especialmente latente en el contexto de los campos de Ciencia, Tecnología, Ingeniería y Matemáticas sobre todo a nivel universitario y, por tanto, laboral. Motivar a las mujeres a estudiar ingeniería es uno de los objetivos que se pueden encontrar en los planes estratégicos no solo de grandes empresas tecnológicas sino también de organizaciones, instituciones y gobiernos. La incorporación de la perspectiva de género en los currículos de educación superior se considera un factor clave a la hora de que los jóvenes comprendan la realidad social. Bajo este marco surge el proyecto de innovación docente cuyo objetivo es incorporar la perspectiva de género en el currículo del Grado en Ingeniería Informática de la Universidad de Salamanca. En el presente trabajo se describe la primera fase del proyecto, un caso de estudio en una asignatura de carácter obligatorio, Ingeniería de Software I.
\end{abstract}

Palabras clave: Ingeniería del Software, Género, Brecha de Género, Desigualdad

Abstract- Gender inequality is a global problem, but is especially latent in the context of the fields of Science, Technology, Engineering and Mathematics, especially at the university level and, therefore, labor. Motivating women to study engineering is one of the objectives that can be found in the strategic plans not only of large technology companies but also of organizations, institutions and governments. The incorporation of the gender perspective in higher education curricula is considered a key factor when young people understand the social reality. In this context emerges the project of teaching innovation whose goal is to incorporate the gender perspective in the curriculum of the Degree in Computer Sciences of the University of Salamanca. This paper describes the first phase of the project, a case study in a compulsory subject, Software Engineering I.

Keywords: Software Engineering, Gender, Gener Gap, Inequality

\section{INTRODUCCIÓN}

Las mujeres representan el $50 \%$ de la población que consume tecnología, pero este porcentaje no se ve representado en las empresas que la desarrollan. A nivel mundial tan solo un $25 \%$ de los empleos tecnológicos están ocupados por mujeres según el último informe del National Center for Women Information Technology (NCWIT) (Ashcraft, McLain, \& Eger, 2016). Esta cifra se reduce al 18\% en España, según datos del gobierno.

El informe "The future of jobs: Employment, skills and workforce strategy for the fourth industrial revolution" presentado en enero de 2016 en la reunión anual del Foro Económico Mundial En Davos (Suiza) ratifica este porcentaje en su capítulo sobre la brecha de género en la industria, donde pone en relieve que a medida que las fuerzas de mercado transforman las industrias en favor de las habilidades tecnológicas, solo un $26 \%$ de los puestos de trabajo del sector de la tecnología los desempeñan mujeres (World Economic Forum, 2016).

En los últimos años, empresas como Google han implementado medidas para reducir la falta de diversidad a la hora de contratar. En cuestiones de género y tecnología, el primer informe realizado el 2014 reveló que tan solo un 17\% de sus empleados del sector tecnológico eran mujeres. Actualmente esa brecha se ha reducido en un $2 \%$ como se puede ver en los resultados de su último informe (https://www.google.com/diversity).

La baja participación de mujeres en estudios de Ciencia, Tecnología, Ingeniería y Matemáticas (STEM) se ha identificado como uno de los principales problemas que hay que resolver para cerrar la brecha de género que existe en el sector tecnológico. Aunque las mujeres han logrado avances importantes en su participación en la educación superior, todavía están poco representadas en estos campos. Este problema es más agudo en los niveles senior en las jerarquías académicas y profesionales.

Si se observan las cifras del último informe Datos y cifras del sistema universitario español correspondiente al curso 2015-2016, publicado por el Ministerio de Educación, Cultura y Deporte, el 54,1\% del total de estudiantes universitarios son mujeres (Ministerio de Educación Cultura y Deporte, 2016). La proporción de mujeres se incrementa entre los titulados universitarios hasta situarse en el 58\% de media. La presencia 
de la mujer es mayoritaria en todas las ramas, con excepción de las titulaciones técnicas. El porcentaje más alto de mujeres, un $69,4 \%$, está en la rama de Ciencias de la Salud y el más bajo, un 25,8\%, en Ingeniería y Arquitectura. Estas cifras se han mantenido estables en los últimos 10 años si se comparan los resultados publicados desde el curso 2005/2006 (http://www.mecd.gob.es/educacion-mecd/areaseducacion/universidades/estadisticas-informes/datoscifras.html).

Los estereotipos sociales en la ingeniería quedan patentes en un concepto o idea falsa de la ingeniería como una profesión masculina que dificulta la identificación de mujeres con ese oficio (Díaz Lucas, 2016; Grass Ramírez, Collazos, \& González González, 2016). Diversos estudios sobre la materia también coinciden en que hay una ausencia de modelos femeninos en la tecnología (Alcalá Cortijo et al., 2005; Barnard, Hassan, Bagilhole, \& Dainty, 2012; Kim, Fann, \& Misa-Escalante, 2011).

Según Buquet Corleto (2011) la incorporación de los estudios de género en los currículos es considerada como un factor que favorece el proceso de institucionalización de la perspectiva de género en las instituciones de educación superior y se enfoca en dos objetivos con distinto alcance, pero complementarios:

- Impacta de manera directa en la preparación académica de las y los jóvenes en proceso de formación al proporcionarles nuevos elementos teóricos y metodológicos para la comprensión de la realidad social.

- Por otro lado, la importancia de la discusión en clase de los temas con perspectiva de género aporta a la formación de las y los jóvenes universitarios elementos para la deconstrucción de las diversas formas de discriminación imperantes en nuestras sociedades y les transmite valores de equidad y respeto a las diferencias.

Bajo este marco surge el proyecto de innovación docente cuyo fin es incorporar la perspectiva de género en el currículo del Grado en Ingeniería Informática de la Universidad de Salamanca (USAL). Para ello se ha planteado como primera fase un caso de estudio en una asignatura de carácter obligatorio, Ingeniería de Software I.

En las siguientes secciones se presenta el contexto en el que se ha desarrollado el proyecto de innovación (2), las actividades llevadas a cabo (3), los resultados obtenidos (4) y por último las conclusiones.

\section{CONTEXTO}

En 2008 la Ley Orgánica 4/2007, de 12 de abril, estableció para la para la Igualdad efectiva de Mujeres y Hombres, que "Las universidades contarán entre sus estructuras de organización con unidades de igualdad para el desarrollo de las funciones relacionadas con el principio de igualdad entre mujeres y hombres”.

Como consecuencia de dicha Ley, en 2008 la Universidad de Salamanca crea la Unidad de Igualdad con el objeto de impulsar el proceso de creación de consenso sobre política universitaria de igualdad, permitir el seguimiento del Plan de Igualdad entre mujeres y hombres de la Universidad de Salamanca, así como velar por el cumplimiento de las normas vigentes. Fue una de las primeras en crearse y además se convirtió en la primera definida en los Estatutos de una Universidad española.

Además de los artículos que regulan la Unidad de Igualdad, cabe destacar que los Estatutos recogen en su artículo $1^{\circ}$ que "las actuaciones de la Universidad están inspiradas en los principios de democracia, igualdad, justicia y libertad. Cualquier forma de sexismo o de discriminación, directa o indirecta, por razón de sexo, va en contra tanto de esos principios como de nuestra sensibilidad y convicciones".

En este contexto, la Unidad de Igualdad ha llevado a cabo durante el curso 2016/2017 el “Análisis de las asignaturas sobre género impartidas por la USAL y las competencias en materias de igualdad de género” (Unidad de Igualdad, 2017). Este estudio pone de manifiesto que en el grado de Ingeniería Informática la única referencia sobre género que aparece en las guías académicas es que "se tendrán en cuenta los derechos fundamentales y de igualdad de oportunidades entre hombres y mujeres, los principios de igualdad de oportunidades”.

El objetivo general de este trabajo ha sido incorporar la perspectiva de género en la docencia de Ingeniería del Software I como la primera fase para introducir en cursos académicos posteriores la perspectiva de género en el Grado de Ingeniería Informática.

Para la consecución del objetivo general planteado se han definido los siguientes objetivos específicos:

1. Incorporar actividades que promocionen la perspectiva de género en el contexto de la Ingeniería de Software.

2. Realizar un estudio mixto sobre la percepción de los/as estudiantes acerca de la brecha de género en contextos tecnológicos.

3. Ofrecer materiales que tenga en cuenta la perspectiva de género.

La asignatura de Ingeniería del Software I se trata de una materia de carácter obligatorio que se imparte en el segundo cuatrimestre de $2^{\circ}$ curso del Grado de Ingeniería Informática. Debido al número de estudiantes la asignatura se divide en dos grupos de teoría, $\mathrm{A}$ y $\mathrm{B}$, siendo el grupo $\mathrm{A}$ en el que se ha llevado a cabo el caso de estudio. Este grupo tiene matriculados 72 estudiantes de los cuáles 60 cursan la asignatura por primera vez (83.33\%), 4 es la segunda vez que se matriculan (5.55\%), 3 es la tercera vez $(4.17 \%)$, otros 3 es la cuarta vez (4.17\%) y 2 están en su quinta matrícula (2.78\%). El 13.89\% son mujeres (10 estudiantes), todas ellas de primera matrícula.

Existen dos modalidades para cursar la asignatura. Una modalidad con un enfoque más tradicional hacia una evaluación final pensada para aquellos/as estudiantes que han cursado la asignatura anteriormente o que no pueden asistir a las clases presenciales por motivos laborales o por solapamiento con otras asignaturas. $\mathrm{Y}$ una modalidad de evaluación continua que implica la asistencia a las sesiones de teoría y práctica y la realización de ejercicios, talleres, presentaciones orales, entregas parciales de la práctica final, etc. Además, los/as estudiantes de años anteriores pueden guardar la nota del trabajo final. 63 estudiantes (87.5\%) han cursado la modalidad de evaluación continua y 65 han entregado el trabajo final (90.28\%). 


\section{DESCRIPCIÓN}

El proyecto se ha dividido en dos grupos de tareas bien diferenciados. En primer lugar, la inclusión de la perspectiva de género en las diferentes actividades que se llevan a cabo en la modalidad de evaluación continua de la asignatura. En segundo lugar, la evaluación de las medidas planteadas para visibilizar la brecha de género.

\section{A. Impacto del proyecto}

Para llevar a cabo la evaluación de las medidas implantadas se han definido dos instrumentos, un pretest y un postest de único grupo (sin control) que posteriormente se analizarán utilizando un enfoque mixto (cualitativo y cuantitativo) (Creswell \& Piano Clark, 2007).

Ambos instrumentos de evaluación se han aplicado de forma anónima a los/as estudiantes de la asignatura que han decidido participar voluntariamente, tanto hombres como mujeres.

El pretest se ha realizado durante la primera semana de docencia de la asignatura, con objeto de no influir en los resultados del mismo con los contenidos y actividades que han formado parte de la introducción de la perspectiva de género.

El postest se ha realizado una vez finalizadas las actividades relacionadas con la perspectiva de género, coincidiendo con la última semana de docencia de la asignatura.

Las preguntas que conforman el pretest y el postest son en su mayoría de respuesta múltiple pero también hay algunas preguntas abiertas. La elaboración de ambos instrumentos ha combinado preguntas definidas ad-hoc y preguntas basadas en trabajos previos. En particular, en el guion de las entrevistas realizadas en el proyecto "Trayectorias de vida tecnológica y género: factores psicosociales implicados en el acceso a las titulaciones de ingeniería informática” (Gil-Juárez, Feliu, VallLlovera, \& Biglia, 2014), y en el cuestionario utilizado en el proyecto "Fortalecimiento de la Equidad de Género en la Educación superior en Colombia” (Rojas Betancur, Méndez Villamizar, \& Montero Torres, 2013).

La única diferencia entre ambos instrumentos radica en que el postest incorpora al final una serie de afirmaciones relacionadas con la incorporación de la perspectiva de género en la asignatura.

Para poder comparar los resultados se ha incluido un identificador único que el estudiante debía calcular restando a su Número de Identificación Fiscal (NIF) una fecha significativa para él.

Tanto el pretest como el postest se han realizado utilizando la herramienta de Formularios de Google y se encuentran disponibles en el informe técnico (García-Holgado, GarcíaPeñalvo, Mena, \& González, 2017).

La validación de ambos instrumentos se realizará a partir de los resultados obtenidos en esta experiencia con el fin de aplicar los instrumentos validados en el siguiente curso académico.

\section{B. Actividades sobre la perspectiva de género}

Las actividades llevadas a cabo se han centrado en hacer visible el problema existente en el ámbito tecnológico con el objetivo de que los/as estudiantes sean conscientes de la realidad social y laboral a la que se enfrentarán una vez finalicen sus estudios. Destacar la importancia de implicar tanto a hombres como a mujeres en este tipo de acciones.

En primer lugar, durante el desarrollo de la asignatura se ha proporcionado información a los/as estudiantes sobre los problemas de género, con especial hincapié en los relacionados con el ámbito tecnológico, mediante el uso del hashtag \#is1usal17 en Twitter. Aunque en cursos anteriores ya se había utilizado esta red social para proporcionar información relacionada con la asignatura, este año se ha enfocado su uso en dar visibilidad a la brecha de género y se ha embebido en el espacio de la asignatura en el Campus Virtual de la Universidad para lograr que lo vieran el mayor número de estudiantes posible. En la Figura 1 se muestran algunos ejemplos.

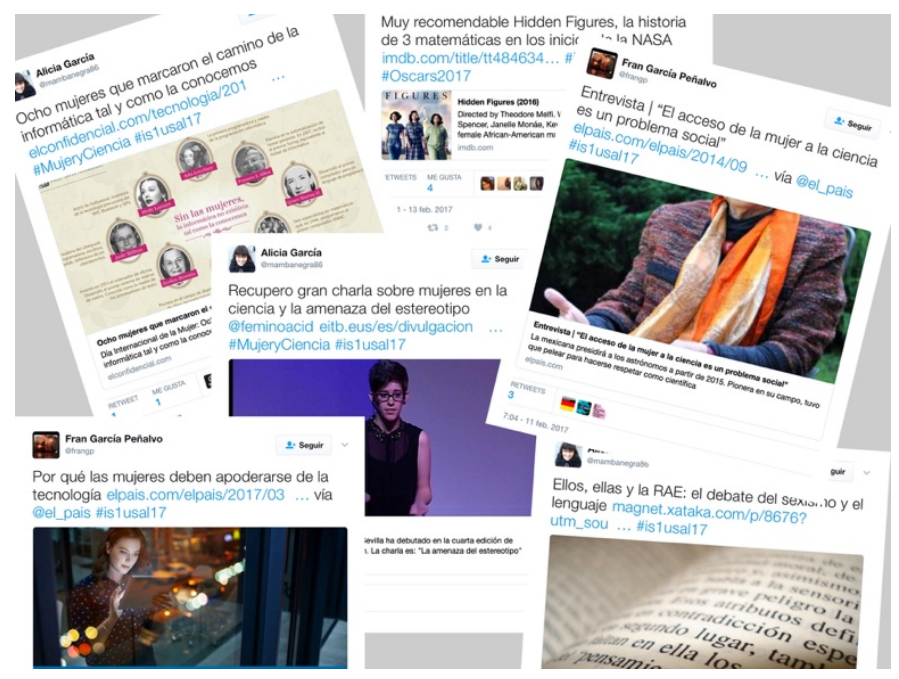

Figura 1. Tweets con el hashtag de la asignatura \#is1usal17

Los materiales utilizados durante las clases, así como aquellos publicados en el Campus Virtual, se han actualizado con el objetivo de utilizar un lenguaje inclusivo que evitara el masculino genérico en la medida de lo posible. A pesar de las indicaciones de la RAE para evitar este tipo de acciones (Bosque, 2012) se ha considerado de especial importancia reflejar a las mujeres de forma explícita en los textos sobre definición y desarrollo de proyectos software.

La asignatura tiene una fuerte componente práctica. A lo largo del cuatrimestre se plantean una serie de problemas a resolver desde el punto de vista de la ingeniería mediante la definición de una herramienta software. Los ejercicios se describen en lenguaje natural y proporcionan los requisitos que debe cumplir el sistema a definir. Todos los ejercicios que se han planteado han estado relacionados con los problemas de género en el ámbito tecnológico. Cabe destacar tres:

- Una aplicación web que proporcione las herramientas necesarias para impulsar la adquisición de competencias STEM en la educación primaria y secundaria con especial énfasis en las chicas, intentando reducir la percepción de desigualdad en la elección de estudios universitarios.

- Un portal de empleo para mujeres especializado en informática, telecomunicaciones y tecnología.

- El portal de una asociación cuyo objetivo principal es dar visibilidad a las mujeres en el ámbito tecnológico a través de diferentes proyectos. 
Cada uno de los enunciados se ha acompañado de una introducción sobre el problema a resolver con enlaces a noticias e informes de actualidad.

Tanto los ejercicios mencionados como la práctica o trabajo final se realizan en grupos formados por dos o tres estudiantes. Como parte del proyecto se ha promovido la presencia de mujeres en el mayor número posible de grupos de prácticas teniendo en cuenta las limitaciones existentes (número de mujeres matriculadas en la asignatura). El equipo docente no interviene en la definición de los grupos por lo que lo que se hizo fue indicarlo como sugerencia durante la presentación de la asignatura.

Por último, el trabajo final ha consistido en definir un portal para promover la visibilidad de la mujer en el contexto STEM, proporcionando un espacio donde tuvieran cabida iniciativas, proyectos, asociaciones, instituciones, etc. relacionadas con reducir la brecha de género en el sector tecnológico. A diferencia de años anteriores donde la funcionalidad del sistema software se definía detalladamente, este año se ha dado total libertad con el objetivo de que investigaran sobre la problemática.

Como parte del trabajo, se les ha pedido una breve memoria técnica en las que han elaborado una introducción al dominio del problema, han destacado los aspectos relevantes del desarrollo del trabajo y han planteado una serie de conclusiones.

Finalmente, como actividad complementaria y voluntaria relacionada con el trabajo final, los grupos de prácticas han presentado delante de sus compañeros/as la memoria técnica.

\section{RESUltados}

Para evaluar los resultados del proyecto se ha utilizado un enfoque mixto a través de dos instrumentos de evaluación, un pretest y un postest, cuyo objetivo es determinar si las medidas planteadas para visibilizar la brecha de género han sido efectivas.

Inicialmente el pretest fue respondido en línea por 55 estudiantes pero dichos datos quedaron invalidados cuando el enlace al cuestionario se hizo público en un foro de Internet. No fue posible restringir el acceso únicamente a usuarios de la comunidad universitaria, con el fin de asegurar el anonimato de los participantes. Debido a ello, se volvió a aplicar el mismo instrumento en horario no lectivo, pero en formato impreso para evitar el problema previo. Se obtuvieron únicamente 9 respuestas por lo que los datos obtenidos mediante el instrumento no son relevantes para evaluar los resultados del proyecto.

Ante esta situación se decidió reorientar la finalidad de los instrumentos, de tal forma que esta primera experiencia sirva para validarlos. La aplicación del segundo instrumento también se ha realizado en horario no lectivo y en formato impreso para evitar que se repitiera el problema del pretest. En este caso se obtuvieron 23 respuestas de las cuáles una no es válida porque no se completó la mitad del cuestionario.

Debido a que el postest engloba las preguntas del pretest la validación del segundo instrumento permitirá evaluar también el primero. Dicha validación se llevará a cabo más adelante.

Para poder conocer el impacto del proyecto sin utilizar los instrumentos ya mencionados, se ha optado por realizar un análisis cualitativo de la introducción y las conclusiones de las memorias técnicas de los trabajos finales. La introducción contiene datos sobre el dominio del problema, es decir, sobre la brecha de género lo que puede proporcionar información sobre el nivel de implicación de los estudiantes a la hora de investigar el problema. En cuanto a las conclusiones, durante la corrección de los trabajos se ha detectado de manera informal que la mayoría de los grupos no tenían conocimientos previos de la problemática y consideran importante que se les haya proporcionado información sobre ello.

El análisis de dichos textos se llevará a cabo una vez finalice la segunda convocatoria de la asignatura con el fin de tener la versión final de todos los trabajos.

Respecto a la definición de grupos de prácticas mixtos, el porcentaje de grupos que incorporan una mujer en relación al número de mujeres que participan en la asignatura es del 50\%, es decir, en la asignatura hay 10 mujeres por lo que si cada una formara parte de un grupo diferente eso nos daría el 100\% en este indicador. Hay 4 grupos mixtos y 1 formado íntegramente por mujeres.

Con relación a la última actividad, la presentación oral del trabajo, 5 grupos hablaron frente a sus compañeros sobre la brecha de género y el portal que habían definido para ayudar a reducirla. Todos los grupos presentados datos de fuentes que no se habían proporcionado durante el curso, lo que demuestra un trabajo de investigación.

\section{CONCLUSIONES}

Existe un gran número de iniciativas a nivel nacional e internacional cuyo objetivo es reducir la brecha de género en el ámbito tecnológico (Espino Espino \& González, 2016). Cabe destacar a nivel internacional Women Techmakers (https://www.womentechmakers.com) cuyo objetivo es lograr una mayor diversidad en el sector tecnológico, y los proyectos IEEE Women in Engineering (http://www.ieee.org/membership_services/membership/wome n/index.html) y ACM-W (https://women.acm.org). A nivel nacional, el Campus Tecnológico de la Universidad de Granada para chicas (http://cs4hs.ugr.es), la iniciativa Tech\&Ladies (http://techandladies.com) o la startup social Adalab (http://adalab.es) cuyo objetivo es invertir en mujeres con talento que no han tenido la oportunidad de desarrollar todo su potencial, para que se conviertan en profesionales digitales.

En el contexto educativo, hay un gran número de iniciativas que trabajan desde edades tempranas para despertar el interés por los estudios STEM, en particular existen diversos proyectos de investigación relacionados con introducir el pensamiento computacional en los colegios (Espino Espino \& González González, 2015; García-Peñalvo, 2016).

En la educación superior, el problema no reside en la elección de estudios STEM, por lo que las iniciativas se enfocan en poner de manifiesto la problemática, lograr mayor visibilidad de las mujeres que estudian carreras técnicas, etc.

El presente proyecto ha cambiado la percepción de los/as estudiantes respecto a los problemas de género en el ámbito tecnológico. Todos los/as estudiantes que han entregado las conclusiones del trabajo final han reconocido que no eran 
conscientes de la brecha de género más allá del bajo número de mujeres que hay en el grado de Ingeniería Informática.

El trabajo también presenta algunas carencias que se mejorarán en el próximo curso académico. En particular, se tomarán medidas para obtener una mayor participación en la aplicación de los instrumentos de evaluación.

\section{AGRADECIMIENTOS}

Este trabajo de investigación forma parte del proyecto de innovación y mejora docente "Inclusión de la perspectiva de género en la asignatura de Ingeniería de Software I" (ID2016/084) financiado por la Universidad de Salamanca en el curso 2016/2017.

Los autores quieren dar las gracias al Grupo de Investigación en InterAcción y eLearning (GRIAL) http://grial.usal.es.

\section{REFERENCIAS}

Alcalá Cortijo, P., Bordons, M., García de Cortázar, M. L., Griñón, M., Guil, A., Muñoz, A., . . . Santesmases, M. J. (2005). Mujer y ciencia. La situación de las mujeres investigadoras en el sistema español deficiencia $y$ tecnología. Retrieved from Madrid, Spain: https://icono.fecyt.es/informesypublicaciones/Paginas/M ujer-y-Ciencia-situacion-de-las-mujeres-investigadorasen-el-sistema-espa\%C3\%B1ol-de-ciencia-ytecnologia.aspx

Ashcraft, C., McLain, B., \& Eger, E. (2016). Women in tech: The facts. Retrieved from https://www.ncwit.org/sites/default/files/resources/ncwit _women-in-it_2016-full-report_final-web06012016.pdf

Barnard, S., Hassan, T., Bagilhole, B., \& Dainty, A. (2012). 'They're not girly girls': an exploration of quantitative and qualitative data on engineering and gender in higher education. European Journal of Engineering Education, 37(2), 193-204. doi:1080/03043797.2012.661702

Bosque, I. (2012). Sexismo lingüístico y visibilidad de la mujer. Boletín de Información Lingüística de la Real Academia Española(1), 1-18.

Buquet Corleto, A. G. (2011). Transversalización de la perspectiva de género en la educación superior: Problemas conceptuales y prácticos. Perfiles educativos, 33(SPE.), 211-225.

Creswell, J. W., \& Piano Clark, V. L. (2007). Designing and conducting mixed methods research. Australian and New Zealand Journal of Public Health, 31(4). doi:10.1111/j.1753-6405.2007.00096.x

Díaz Lucas, S. (2016). Promoción Estudios STEM, Ciencia, Tecnología, Ingeniería y Matemáticas, en Navarra. Retrieved from Navarra: https://www.navarra.es/NR/rdonlyres/FEF75753-5C364FEB-8C83-

EDBC9A76C709/365971/PromocionestudiosSTEMcienc iatecnologiaingenieriaym.pdf

Espino Espino, E. E., \& González, C. S. (2016). Educación, mujeres y tecnología: análisis de preferencias formativas según el género. $I+T+C$ Investigación, Tecnología y Ciencia, 91-101.

Espino Espino, E. E., \& González González, C. S. (2015). Estudio sobre diferencias de género en las competencias y las estrategias educativas para el desarrollo del pensamiento computacional. RED-Revista de Educación a Distancia, 46(12). doi:10.6018/red/46/12

García-Holgado, A., García-Peñalvo, F. J., Mena, J., \& González, C. S. (2017). Pretest y postest para evaluar la introducción de la perspectiva de género en la docencia de asignaturas de Ingeniería Informática (Technical Report GRIAL-TR-2017-005). Retrieved from Salamanca, http://repositorio.grial.eu/handle/grial/92

García-Peñalvo, F. J. (2016). Proyecto TACCLE3 - Coding. In F. J. García-Peñalvo \& J. A. Mendes (Eds.), XVIII Simposio Internacional de Informática Educativa, SIIE 2016 (pp. 187-189). Salamanca, España: Ediciones Universidad de Salamanca.

Gil-Juárez, A., Feliu, J., Vall-Llovera, M., \& Biglia, B. (2014). Trayectorias de vida tecnológica y género: factores psicosociales implicados en el acceso a las titulaciones de ingeniería informática. Retrieved from Instituto de la Mujer:

http://www.inmujer.gob.es/areasTematicas/estudios/estu dioslinea2014/docs/Trayectorias_vida_tecnologica_gener o.pdf

Grass Ramírez, B. E., Collazos, C. A., \& González González, C. S. (2016). Diferencias de género en programas de informática: estudio de caso colombiano. In L. Moreno López, E. J. Rubia Cuestas, V. M. R. Penichet, \& F. J. García-Peñalvo (Eds.), Actas del XVII Congreso Internacional de Interacción Persona-Ordenador Interacción 2016. 14-16 de septiembre de 2016, Salamanca, España (pp. 249-253). Salamanca, Spain: Ediciones Universidad de Salamanca.

Kim, K. A., Fann, A. J., \& Misa-Escalante, K. O. (2011). Engaging Women in Computer Science and Engineering: Promising Practices for Promoting Gender Equity in Undergraduate Research Experiences. Trans. Comput. Educ., 11(2), 1-19. doi:10.1145/1993069.1993072

Ministerio de Educación Cultura y Deporte. (2016). Datos y cifras del sistema universitario español. Curso 2015/2016. Retrieved from Madrid, Spain: http://www.mecd.gob.es/dms/mecd/educacionmecd/areas-educacion/universidades/estadisticasinformes/datos-cifras/datos-y-cifras-SUE-2015-16-web.pdf

Rojas Betancur, M., Méndez Villamizar, R., \& Montero Torres, L. (2013). Satisfacción laboral y relaciones de género en la Universidad. Revista Virtual Universidad Católica del Norte(40).

Unidad de Igualdad. (2017). Análisis de las asignaturas sobre género impartidas por la USAL/Competencias en materias de igualdad de género. Retrieved from Unidad de Igualdad, University of Salamanca: https://igualdadefectivablog.files.wordpress.com/2017/05 /asignaturas-sobre-gecc81nero-impartidas-por-la-usal.pdf

World Economic Forum. (2016). The future of jobs: Employment, skills and workforce strategy for the fourth industrial revolution. Retrieved from http://www3.weforum.org/docs/WEF_Future_of_Jobs.pd $\mathrm{f}$ 